\title{
Improving Outcomes in Perihilar Cholangiocarcinoma
}

\author{
Karl J. Oldhafer ${ }^{a, b}$ Thomas von Hahn ${ }^{b, c}$ Marianna Arvanitakis ${ }^{d}$ \\ Masato Nagino ${ }^{e}$ Orlando J.M. Torres ${ }^{f}$ \\ a Division of Hepatobiliary and Pancreatic Surgery, Department of Surgery, Asklepios Hospital Barmbek,

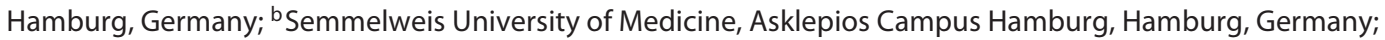 \\ 'Department of Gastroenterology and Hepatology, Asklepios Hospital Barmbek, Hamburg, Germany; \\ ${ }^{\mathrm{d}}$ Department of Gastroenterology, Hepatopancreatology and Digestive Oncology, CUB Hôpital Erasme, \\ Université Libre de Bruxelles, Brussels, Belgium; ${ }^{\mathrm{e}}$ Aichi Cancer Center, Nagoya, Japan; ${ }^{\mathrm{f}}$ Hepatopancreatobiliary Unit, \\ Department of Gastrointestinal Surgery, Hospital Universitário - School of Medicine, Federal University \\ of Maranhão, Maranhão, Brazil
}

\section{Oldhafer/von Hahn: Is Preoperative Bismuth- Collette Staging of Perihilar Cholangiocarcinoma Important when Planning Resection?}

\section{Arvanitakis}

High-quality cross-sectional imaging before resection is the single most crucial step in the diagnostic algorithm. Depending on institutional expertise, this may be accomplished with thin-slice, high-resolution computed tomography (CT) or magnetic resonance imaging/magnetic resonance cholangiopancreatography (MRI/MRCP) [1]. Whereas CT with contrast is highly accurate in determining vascular invasion, local lymph nodes and distant metastasis, MRI with MRCP provide a clearer delineation of the intrahepatic extension of the tumor within the bile ducts, and therefore a precise staging according to Bismuth-Collette [2]. The above staging describes the extent to which the common hepatic duct, duct confluence, and left and right ducts are involved by tumor. Although this classification is important to determine the operative strategy (tumor local excision with or without extended liver resections), it should be combined with assessment of vascular involvement, lobar atrophy, the presence of the nodal and extraregional metastasis, and clinical information such as the presence of jaundice and performance status when planning resection [3]. Finally, a multidisciplinary approach and discussion are indispensable to optimize management.

\section{Nagino}

As you know, Bismuth classification is not "staging" system, just indicating the main tumor location. Operative planning should be made considering tumor extension, individual anatomy, vascular involvement, liver function, etc. Bismuth classification is widely disseminated in the world, and useful to understand the tumor location, but if I'm forced to say, it is not so important when planning resection.

\section{Torres}

Yes, I do routinely.

\section{Oldhafer/von Hahn: Do you See a Role for Cholangioscopy in the Diagnostic Workup?}

\section{Arvanitakis}

Single operator cholangioscopy can allow direct visualization of the biliary epithelium and targeted biopsies of biliary lesions thereby increasing the diagnostic yield of tissue sampling in patients with indetermined strictures, to avoid unnecessary surgical resection. For visual impression and targeted tissue biopsies, previous meta-analysis demonstrated, respectively, a sensitivity of 90 and $60 \%$ with a specificity of 80 and $98 \%[4,5]$. These values will probably improve with the digital version of the cholangioscope, which provides higher quality imaging [6], as 
well as with the use of defined terminology [7]. Furthermore, in selected cases, cholangioscopy can provide information on the tumor extension of extrahepatic cholangiocarcinoma, to define the type of resection [8]. Nevertheless, cholangioscopy requires access to the biliary tract through ERCP and may lead to cholangitis; therefore, careful weighting of advantages and risks is important.

\section{Nagino}

No! Surely the most up-to-date cholangioscopy may offer beautiful images, leading to good understanding of tumor nature, especially for papillary tumor. Cholangioscopy is not necessary for invasive infiltrating tumor which is predominant in perihilar CC. I think it is less likely cholangioscopy can increase diagnostic accuracy of tumor extension, and, in turn, improve patient survival after resection.

\section{Torres}

No, we have SpyGlass only in the private hospital. Then, we decided to use it selectively, for patients that we need biopsy for better definition of surgery. In the public hospital, we do not have SpyGlass.

\section{Oldhafer/von Hahn: How Is Your Timing of MRCP in the Diagnostic Workup?}

\section{Arvanitakis}

As mentioned earlier, MRI and MRCP is an important tool for staging. MRCP should be performed before any endoscopic or radiological intervention because the presence of stents can hinder correct interpretation of imaging $[1,3,9,10]$. Furthermore, MRCP is crucial to plan biliary drainage if required, after discussion in a multidisciplinary setting.

\section{Nagino}

I prefer MDCT rather than MRI. In Nagoya University, MRI is not performed in most cases.

\section{Torres}

At the beginning of the evaluation.

\section{Oldhafer/von Hahn: Is a Bismuth-Corlette Stage IV Patient Still Non-Resectable?}

\section{Arvanitakis}

Currently, contraindications for surgical resection in patients with hilar cholangiocarcinoma include: (1) bilateral tumor extension involving left and right secondary biliary radicles (Bismuth-Corlette stage IV); (2) unilobar involvement with encasement of contralateral portal vein or hepatic artery; (3) bilateral vascular involvement; (4) distant metastases; (5) underlying liver disease (advanced fibrosis, cirrhosis); (6) future liver remnant $<20-30 \%$ and no or poor response to portal vein occlusion; and (7) severe comorbidities [11]. Indeed, patients with BismuthCorlette stage IV have advanced disease, and extensive resection is required to obtain an R0 margin. This is reflected in poor surgical outcome, characterized by high morbidity and mortality (76 and 19\%, respectively), as well as low 5-year survival rates reported in previous series [12]. Nevertheless, in well-selected candidates, recent publications show an improvement in outcome when surgery is performed in expert centers [13]. This remains to be confirmed. Another emerging surgical approach that can be discussed in these patients is liver transplantation used in combination with neoadjuvant chemoradiotherapy [3].

\section{Nagino}

No! This concept is out of date $[14,15]$.

\section{Torres}

No. In selected cases, stage IV is resectable after MDT evaluation.

\section{Oldhafer/von Hahn: To What Extent Do You Recommend Preoperative Biliary Drainage in Potentially Resectable Perihilar Cholangiocarcinoma?}

\section{Arvanitakis}

Jaundice is a typical manifestation of perihilar cholangiocarcinoma. The aim of biliary drainage in resectable disease should be decided in the setting of a multidisciplinary team. There is clear consensus that preoperative biliary decompression is indicated in patients with cholangitis, patients undergoing preoperative anti-neoplastic therapy, patients with hyperbilirubinemia-induced malnutrition, hepatic insufficiency, or renal insufficiency. It is also indicated when estimated future liver remnant volume is $<30 \%$ and portal vein embolization (PVE) is needed to obtain hypertrophy of the remnant liver after surgery. In this case, the remnant liver should be drained, contralateral to the PVE segment $[3,10]$. Biliary drainage can be provided endoscopically or percutaneously. Risks related to biliary drainage include cholangitis and seeding. In our center, selective biliary drainage is performed when necessary by inserting plastic stents and avoiding injecting contrast in segments which are not drained.

\section{Nagino}

In principle, preoperative biliary drainage by ENBD or in-stent should be indicated in ALL patients who have obstructive jaundice. 
Biliary drainage is unnecessary in patients whose bilirubin level is less than $5 \mathrm{mg} / \mathrm{dL}$ at first visit and who are to undergo left, not right, hemihepatectomy. However, the waiting period is longer than 1 month in most highvolume centers. Considering this, biliary drainage is mandatory as preoperative management [16].

\section{Torres}

Less than $40 \%$ of FLR, biliary sepsis (cholangitis), severe/long-lasting jaundice, hyperbilirubinemia-induced malnutrition, renal insufficiency, patients undergoing PVE.

\section{Oldhafer/von Hahn: How Do You Follow Up Patients after Curative Resection of Cholangiocarcinoma?}

\section{Arvanitakis}

The initial site of recurrence following resection is more likely to be locoregional in patients with hilar cholangiocarcinoma (59\%) [3]. Therefore, cross-sectional imaging, and especially MRI and MRCP are proposed for follow-up. Diffusion-weighted imaging can help in detecting recurrence. On the other hand, imaging by FDGPET lacks the sensitivity and specificity required as hilar cholangiocarcinomas tend not to be FDG avid.

\section{Nagino}

In principle, for the first 5 years, laboratory blood exam at 3-4 months' interval, and CT at least 2 times per year. After 5 years, laboratory blood exam at 6 months' interval, and one CT per year. Usually we follow up patients for 10 years after surgery [17].

\section{Torres}

Clinical evaluation, image MRI, tumor markers.

\section{Conflict of Interest Statement}

Karl J. Oldhafer, Thomas von Hahn, Marianna Arvanitakis, Masato Nagino: no conflicts of interest to declare. Orlando J.M. Torres: I certify that I have NO affiliations with or involvement in any organization or entity with any financial interest in the subject matter or materials discussed in this article.

\section{Participants}

Prof. Dr. med. Karl J. Oldhafer

Department of Surgery, Division of HBP Surgery

Asklepios Hospital Barmbek

Rübenkamp 220

22307 Hamburg, Germany

k.oldhafer@asklepios.com

Prof. Dr. med. Thomas von Hahn

Department of Surgery, Division of HBP Surgery

Asklepios Hospital Barmbek

Rübenkamp 220

22307 Hamburg, Germany

t.hahn@asklepios.com

Prof. Marianna Arvanitakis

Department of Gastroenterology, Hepatopancreatology and

Digestive Oncology

CUB Hôpital Erasme

Université Libre de Bruxelles

Route de Lennik 808

1070 Brussels, Belgium

Marianna.Arvanitaki@erasme.ulb.ac.be

Dr. Masato Nagino

Aichi Cancer Center

Kanokoden, Chikusa-ku

Nagoya 464-8681, Japan

m.nagino@aichi-cc.jp

nagino@med.nagoya-u.ac.jp

Prof. Orlando J.M. Torres

Department of Gastrointestinal Surgery

Hepatopancreatobiliary Unit

University Hospital, Federal University of Maranhao

Rua Barao de Itapari

227 Centro C.E.P. 65

Sao Luis, Maranhao 020-070, Brazil

o.torres@uol.com.br

\section{References}

1 Engelbrecht MR, Katz SS, van Gulik TM, Laméris JS, van Delden OM. Imaging of perihilar cholangiocarcinoma. AJR Am J Roentgenol. 2015 Apr;204(4):782-91.

2 Bismuth H, Corlette MB. Intrahepatic cholangioenteric anastomosis in carcinoma of the hilus of the liver. Surg Gynecol Obstet. 1975 Feb;140(2):170-8.

3 Mansour JC, Aloia TA, Crane CH, Heimbach JK, Nagino M, Vauthey JN. Hilar cholangiocarcinoma: expert consensus statement. HPB (Oxford). 2015 Aug; 17(8):691-9.
4 Sun X, Zhou Z, Tian J, Wang Z, Huang Q, Fan $\mathrm{K}$, et al. Is single-operator peroral cholangioscopy a useful tool for the diagnosis of indeterminate biliary lesion? A systematic review and meta-analysis. Gastrointest Endosc. 2015 Jul; 82(1):79-87.

5 Navaneethan U, Hasan MK, Lourdusamy V, Njei B, Varadarajulu S, Hawes RH. Single-operator cholangioscopy and targeted biopsies in the diagnosis of indeterminate biliary strictures: a systematic review. Gastrointest Endosc. 2015 Oct;82(4):608-14.e2.
6 Gerges C, Beyna T, Tang RS, Bahin F, Lau JY, van Geenen E, et al. Digital single-operator peroral cholangioscopy-guided biopsy sampling versus ERCP-guided brushing for indeterminate biliary strictures: a prospective, randomized, multicenter trial (with video). Gastrointest Endosc. 2020 May;91(5):110513. 
7 Sethi A, Tyberg A, Slivka A, Adler DG, Desai AP, Sejpal DV, et al. Digital Single-operator Cholangioscopy (DSOC) Improves Interobserver Agreement (IOA) and Accuracy for Evaluation of Indeterminate Biliary Strictures: The Monaco Classification. J Clin Gastroenterol. 2020 Feb 7. doi: 10.1097/ MCG.0000000000001321. Online ahead of print.

8 Kanno Y, Koshita S, Ogawa T, Masu K, Kusunose H, Sakai T, et al. Peroral cholangioscopy by SpyGlass DS versus CHF-B260 for evaluation of the lateral spread of extrahepatic cholangiocarcinoma. Endosc Int Open. 2018 Nov; 6(11):E1349-54.

9 Dumonceau JM, Tringali A, Papanikolaou IS, Blero D, Mangiavillano B, Schmidt A, et al. Endoscopic biliary stenting: indications, choice of stents, and results: European Society of Gastrointestinal Endoscopy (ESGE) Clini- cal Guideline - Updated October 2017. Endoscopy. 2018 Sep;50(9):910-30.

10 Tringali A, Boškoski I, Costamagna G. Endoscopic Stenting in Hilar Cholangiocarcinoma: When, How, and How Much to Drain? Gastroenterol Res Pract. 2019 Nov;2019:5161350.

11 Sapisochin G, Ivanics T, Subramanian V, Doyle M, Heimbach JK, Hong JC. Multidisciplinary treatment for hilar and intrahepatic cholangiocarcinoma: A review of the general principles. Int J Surg. 2020 Oct;82S:7781

12 Ito F, Cho CS, Rikkers LF, Weber SM. Hilar cholangiocarcinoma: current management. Ann Surg. 2009 Aug;250(2):210-8.

$13 \mathrm{Ku} \mathrm{D}$, Tang R, Pang T, Pleass H, Richardson A, Yuen L, et al. Survival outcomes of hepatic resections in Bismuth-Corlette type IV cholangiocarcinoma. ANZ J Surg. 2020 Sep;90(9): 1604-14.
14 Ebata T, Mizuno T, Yokoyama Y, Igami T, Sugawara G, Nagino M. Surgical resection for Bismuth type IV perihilar cholangiocarcinoma. Br J Surg. 2018 Jun;105(7):829-38.

15 Ebata T, Kosuge T, Hirano S, Unno M, Yamamoto M, Miyazaki M, et al. Proposal to modify the International Union Against Cancer staging system for perihilar cholangiocarcinomas. Br J Surg. 2014 Jan;101(2):79-88.

16 Maeda T, Ebata T, Yokoyama Y, Mizuno T, Yamaguchi J, Onoe S, et al. Preoperative course of patients undergoing endoscopic nasobiliary drainage during the management of resectable perihilar cholangiocarcinoma. J Hepatobiliary Pancreat Sci. 2019 Aug;26(8):341-7.

17 Nakahashi K, Ebata T, Yokoyama Y, Igami T, Mizuno T, Yamaguchi J, et al. How long should follow-up be continued after R0 resection of perihilar cholangiocarcinoma? Surgery. 2020 Oct;168(4):617-24. 